\title{
Gebäudeautomation als wichtiger Puzzlestein auf dem Weg zur Smart City
}

\author{
D. Bruckner OVE， D. Dietrich OVE，F. Kupzog OVE
}

Online publiziert: August 23, 2012

(c) Springer-Verlag 2012

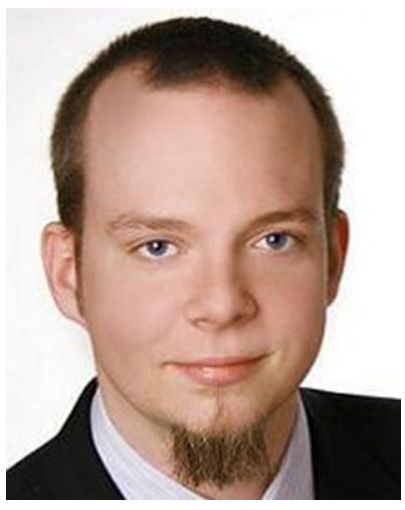

Univ.-Ass. Dipl.-Ing. Dr. Dietmar Bruckner

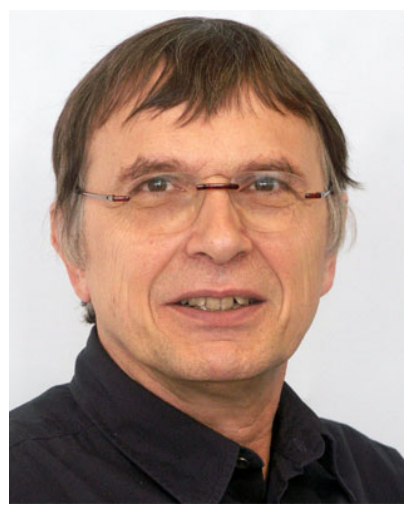

O. Univ.-Prof. Dipl.-Ing. Dr. Dietmar Dietrich

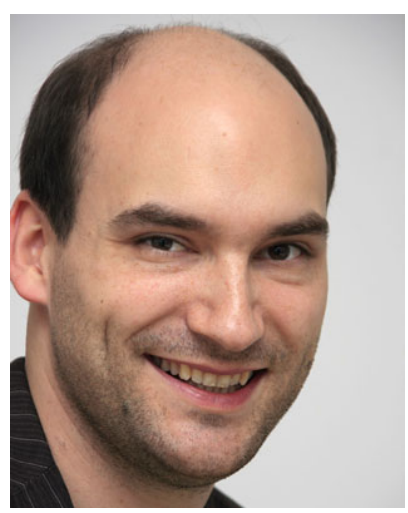

Univ.-Ass. Dipl.-Ing. Dr. Friederich Kupzog
Umweltschutz, Klimawandel, Abhängigkeit von Energieimporten und der steigende Preis fossiler Energieträger haben in den letzten Jahren zu einer weltweiten Debatte über eine radikale Umgestaltung der Energiesysteme geführt. In vielen europäischen Ländern liegt neben der Reduzierung des Energieverbrauchs und der Effizienzsteigerung bei der Energiegewinnung der Fokus auf einer signifikanten Erhöhung des Energieanteils aus erneuerbaren Quellen. Dänemark hat seinen Anteil an Energiegewinnung aus Windkraft extrem erhöht. Deutschland verzichtet auf Atomkraftwerke und Spanien nutzt große Flächen zur Gewinnung von Solarenergie. Aufgrund der beliebigen Energieeinspeisung aus Wasserkraftwerken, vor allem aber aus Wind- und Solaranlagen, ist die Integration dieser Quellen in die bestehenden, über viele Jahrzehnte langsam gewachsenen Energiesysteme im Allgemeinen problematisch. Es ist verständlich, dass die Steuerung und Regelung solch komplexer Prozesse weit über die klassische Energietechnik hinausgeht und nun zunehmend andere wissenschaftliche Bereiche miteinbezogen werden müssen.

In dieser Ausgabe von e\&i möchten wir Ihnen ausgewählte Bereiche vorstellen, in denen die Automatisierungstechnik in der Diskussion zur Umgestaltung der Energiesysteme eine wichtige Rolle einnimmt.
Nach einer Einführung in die Thematik mit dem Aufsatz "Cyber Physical Systems beschreiben neue Energieversorgungssysteme", in dem eine grundlegend neue Denkweise zur Lösung des Problems vorgestellt wird, wenden wir uns zunächst dem Energieverbrauch in Städten zu. Nachdem der Anteil der Weltbevölkerung in Städten den am Land nun auch weltweit, nicht nur in den Industrienationen, überholt hat und die Einwohnerentwicklung in den urbanen Zentren weiterhin stark zunimmt, ist es sinnvoll, den Hebel auf Stadtebene anzusetzen, um die Strukturen so zu verändern, dass eine nachhaltige Versorgung wirtschaftlich, technisch und organisatorisch möglich ist Integration of centralized energy monitoring specifications into the planning process of a new urban development area: a step towards smart cities).

Weniger aus der Sicht der urbanen Zentren als aus Sicht der faktisch vorhandenen Energieversorgungsstrukturen versucht der Ansatz des Smart Grid durch zeitnahes Monitoring und intelligente, koordinierte Steuerung der Energienetzteilnehmer erneuerbare Energieträger in die bestehenden Strukturen zu integrieren. Dies geschieht unter massivem Einsatz von Informations- und Kommunikationstechnologien (Sicherheits- und Datenschutzanforderungen an Smart Grid-Technologien).

Ein signifikanter Anteil des urbanen Energieverbrauchs liegt im Bereich großer funktionaler Gebäude. Für die Realisierung effizienter Energiesysteme spielen die Nutzbarmachung der Effizienzsteigerungspotentiale in diesem Bereich sowie die Abstimmung auf gebäudeintegrierte und externe erneuerbare Energieträger eine große Rolle. Hier liegt auch ein großer Schwerpunkt österreichischer Smart Grid-Forschung (Energy aware building automation enables Smart Grid-friendly buildings).

Zentrales Element bei der Nutzbarmachung der Ressource Gebäude mit ihren Lastverschiebepotentialen im Netz ist die Kopplung klassischer Gebäudeautomation mit den im Entstehen befindlichen IT-Infrastrukturen der Smart Grids. Einen Einblick in aktuelle Entwicklungen im Bereich der Gebäudeautomation bietet der Beitrag Current developments and challenges in building automation.

Die Automation von Gebäuden spielt also eine wichtige Rolle bei der Abstimmung des Endverbrauchs mit variabler Einspeisung im intelligenten Energiesystem der Zukunft. Dies kann bis in den Heimbereich weitergedacht werden. Nicht zuletzt in diesem Bereich ist eine Anbindung an das Smart Grid aber nur dann denkbar, wenn dadurch auch Zusatznutzen für den Endverbraucher entsteht (Wissensrepräsentation für das adaptive Eigenheim im Kontext von Smart Cities).

Viele der angedachten Technologien im Wohnbereich, sei es im Sinne effizienter Energienutzung oder auch im Sinne von betreuten

Bruckner, Dietmar, Univ.-Ass. Dipl.-Ing. Dr., Institut für Computertechnik, Technische Universität Wien, Wien, Österreich (E-Mail: bruckner@ict.tuwien.ac.at); Dietrich, Dietmar, 0. Univ.-Prof. Dipl.-Ing. Dr., Institut für Computertechnik, Technische Universität Wien, Wien, Österreich; Kupzog, Friederich, Univ.-Ass. Dipl.-Ing. Dr., Institut für Computertechnik, Technische Universität Wien, Wien, Österreich 
Wohnkonzepten wie Ambient Assisted Living, beruhen auf Sensorinformationen aus dem privaten Lebensumfeld. Noch immer sind die Kosten für solche Sensornetzwerke relativ hoch, was sich negativ auf die Nutzbarmachung von innovativen Anwendungen auswirkt. Der Beitrag Relieved commissioning and human behavior detection in Ambient Assisted Living Systems beschäftigt sich mit dieser Her- ausforderung und damit, wie man den Konfigurationsaufwand und somit die Anschaffungskosten solcher Systeme möglichst gering halten kann.

Wir wünschen Ihnen eine spannende und aufschlussreiche Lektüre. 